\title{
Mesure de l'activité des saponines de la luzerne par les larves du ver de farine: Tenebrio molitor L. (Coléoptère, Tenebrionidae). II. — Recherche des fractions de saponines responsables des effets antinutritionnels observés
}

Pascale PRACROS

avec la collaboration technique de Christine Couranjou

I.N.R.A., Station de Zoologie, Centre de Recherches de Bordeaux, F 33140 Pont-de-la-Maye

Les saponines de certaines variétés de luzerne sont à l'origine d'un effet antinutritionnel qui peut être observé lors d'essais d'alimentation du poulet et, également, du ver de farine, Tenebrio molitor. La recherche des fractions responsables de l'activité de ces substances ingérées par l'insecte et la mise au point de méthodes de dosage par HPLC ont permis d'étudier les relations entre la teneur en saponines des régimes alimentaires proposés, la composition de ce mélange de substances (teneur en génines totales, en acide médicagénique et en acide luzernique) et leur activité antinutritionnelle.

Pour cela, 2 types d'essai ont été mis en place:

La comparaison des performances des larves de Tenebrio molitor élevées dans des régimes expérimentaux contenant pour unique source de protéine des poudres de feuilles de 41 variétés de luzerne, aux teneurs en saponines, génines, acides médicagénique et luzernique déterminées par la technique de chromatographie liquide haute performance au laboratoire de pharmacognosie de l'université de Reims, montre que les larves sont sensibles aux saponines et plus particulièrement à celles qui ont pour génine l'acide médicagénique, et que pour les variétés à teneur en acide médicagénique faible, le taux d'acide luzernique permet d'expliquer de façon significative le classement des variétés de luzerne par l'insecte.

Une seconde expérimentation, consistant en l'incorporation de saponines dans un régime alimentaire standardisé, a permis de vérifier l'effet de ces substances et de certaines de leurs fractions sur les performances de l'insecte. Cet essai montre que les traitements subis par les diverses molécules tout au long des étapes de purification n'altèrent en rien leurs propriétés antinutritionnelles et indique que les larves sont sensibles aux saponines purifiées dès le seuil de 0,4 p. 100 de régime alimentaire sec en présence de 1 p. 100 de cholestérol.

Mots clés additionnels : Substances allélochimiques, acide médicagénique, acide luzernique. Tenebrio molitor L. II. - Search for saponins with antinutritional activity.

The saponin content of some lucerne varieties is responsible for the poor nutritional value of lucerne for chick, but also for larvae of the yellow mealworm Tenebrio molitor, which is taken as a biological indicator of saponin activity. Investigation of fractions responsible for the activity and analytical assays have allowed study of the relationship between activity and composition of the mixtures. The "yellow mealworm test" was applied to leaves of 41 varieties of lucerne. The saponin content of these leaves were determined by High Performance Liquid Chromatography analysis. Results obtained with the yellow mealworm test showed that the larvae were sensitive to saponins, and especially to those with medicagenic acid as the genin. A good correlation was also obtained between the feeding and growth of the insects and the content of lucernic acid, for lucerne varieties with a low medicagenic acid content. A second method of testing, by incorporation of purified saponins into a standardized diet, gave two main results : 1) insect feeding and growth were disturbed by an incorporation rate of $0.4 \%$ of saponins in the diet ; 2) purification did not depress the biological activity of the saponins. 


\section{INTRODUCTION}

Les saponines sont les substances que les auteurs rendent responsables des effets antinutritionnels observés chez les volailles lors de l'utilisation de poudre de luzerne pour leur alimentation (COONEY et al., 1948; ANDERSON, 1957; HEYWANG et al., 1959). Leur dosage dans les variétés de luzerne est donc d'une grande importance.

Les saponines sont des hétérosides résultant de la liaison entre une partie aglycone, la génine, et un ou plusieurs sucres. Les génines des saponines de la luzerne (Medicago sativa L.) sont des triterpènes, unités à 30 atomes de carbone. La formule de la génine majoritaire des saponines des feuilles de luzerne fut établie par DJERASSI et al. (1957) qui la baptisa acide médicagénique. En 1959, Livingston isole une deuxième génine, l'acide luzernique qui ne fut identifiée que récemment (Guillaume, 1986). Actuellement, les génines de la luzerne dont on connait la structure sont au nombre de 8 ; ce sont les acides médicagénique, luzernique et zahnique, la bayogénine, l'hédéragénine, les soyasapogénols $\mathrm{A}$ et $\mathrm{B}$ et un composé nouveau mis en évidence par Guillaume (1986).

L'identification des saponines passe par la caractérisation des génines puis par la détermination de la nature des sucres qui y sont fixés. De nombreuses méthodes d'hydrolyse (méthodes enzymatiques ou chimiques) qui permettent de séparer les saponines en leurs 2 unités ont été décrites (GESTETNER, 1971 ; GOLDSTEIN et al., 1965). Ces méthodes destructives nécessitent de grandes quantités d'un matériel précieux obtenu après de multiples étapes de purification. Les travaux de LAvaud (1986) ont abouti à la mise au point d'une méthode analytique globale qui n'altère pas la saponine étudiée et en donne la structure sans ambiguité par l'utilisation de la spectrométrie de résonance magnétique nucléaire (RMN) (MASSIOT et al., 1986).

La teneur en acide médicagénique des feuilles, tiges ou racines de luzerne peut être déterminée par chromatographie gazeuse (RAO \& BORIES, 1987). L'utilisation de la technique de chromatographie liquide haute performance (HPLC) est à l'origine du dosage des saponines totales, des génines totales et de deux d'entre elles (les acides médicagénique et luzernique) réalisé par Guillaume (1986) à partir de feuilles et de concentrés protéiques de luzerne. Cinq saponines ont pu être séparées et leurs structures moléculaires ont été déterminées; elles ont reçu les noms de GCD 20 (fig. 1a), GCD 21 (fig. 1b), GCD 23, GCD 24 et GCD 25, par ordre de complexité croissante correspondant à une augmentation du nombre de leurs sucres.

Les travaux entrepris depuis 1985 dans le cadre de la valorisation des protéines foliaires de la luzerne pour l'alimentation des monogastriques ont conduit à l'utilisation d'une méthode de mesure de l'effet antinutritionnel des saponines et de plusieurs génines chez le ver de farine (Tenebrio molitor L.), insecte particulièrement sensible à la présence de ces substances dans son régime alimentaire (PrACros, 1988). L'utilisation de cet insecte test a permis de mesurer l'activité biologique des saponines soit à partir de variétés de luzerne différemment pourvues en ces substances, soit par utilisation d'extraits purifiés puis fractionnés.

\section{MATÉRIEL ET MÉTHODE}

\section{A. Matériel végétal et extraits}

Les feuilles de 41 variétés de luzerne implantées à la station d'amélioration des plantes de Lusignan (I.N.R.A.) au printemps 1985 ont été récoltées en août de cette même année au stade début floraison, desséchées à $80^{\circ} \mathrm{C}$ puis broyées finement (dimension des particules inférieure à $0,5 \mathrm{~mm}$ ). Les poudres ainsi obtenues ont été conditionnées en sachets étanches et transmises aux laboratoires chargés des diverses analyses ou expérimentations réalisées dans le cadre d'un contrat dont l'objectif était la recherche et l'adaptation de méthodes rapides (physiques ou biologiques) de détection des substances antinutritionnelles présentes dans les plantes protéagineuses.

Les analyses, réalisées au laboratoire de pharmacognosie de la faculté de pharmacie de Reims, ont permis d'obtenir les teneurs en saponines totales, en génines totales, en acide médicagénique et en acide luzernique (par HPLC) pour chacune des 41 variétés de luzerne.

Les saponines isolées au laboratoire de Reims selon la méthode décrite par Guillaume (1986) et fournies pour la réalisation du test biologique furent au nombre de 3 : un mélange de saponines extraites de racines de luzerne (A), un mélange de saponines extraites de concentré protéique (PX) de feuilles de luzerne (B) avec un rendement de 5 p. 1000 et le mélange des saponines GCD 20 et GCD 21 (C) purifiées à partir du PX de feuilles de luzerne et dont les structures moléculaires sont connues (fig. 1).

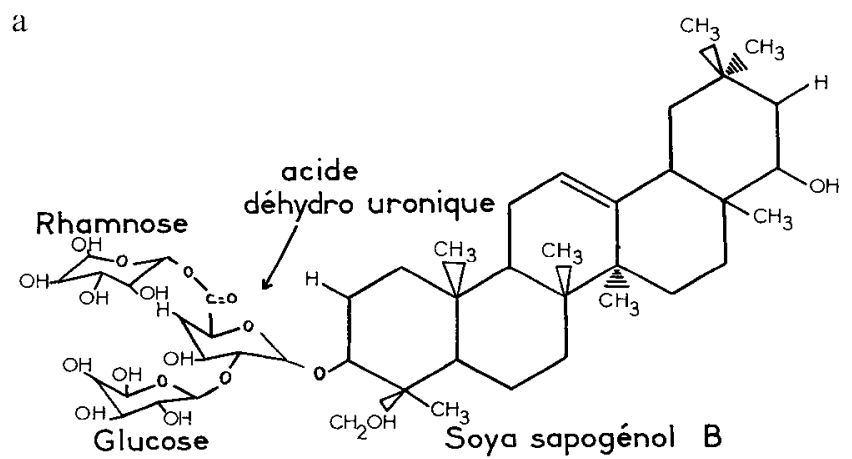

b

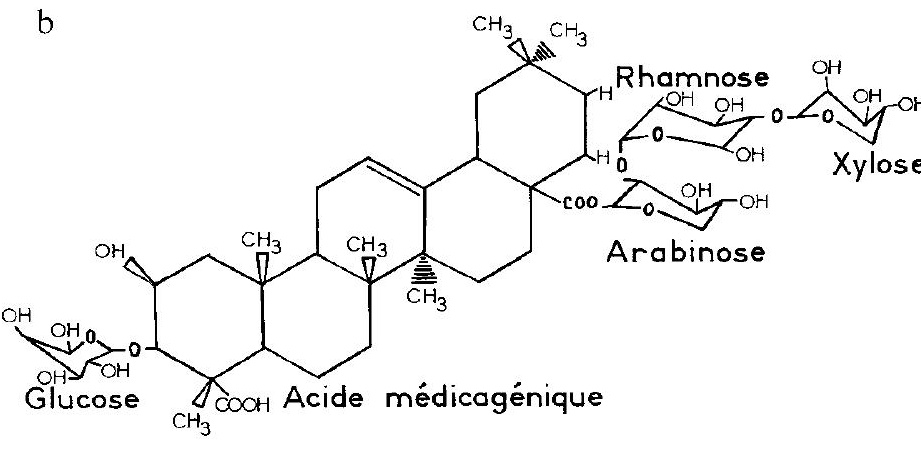

Figure 1

Structures chimiques de 2 saponines de la luzerne : GCD 20 (figure 1a) et GCD 21 (figure $1 \mathrm{~b}$ )

Chemical structures of two lucerne saponins : GCD 20 (figure 1a) and GCD 21 (figure $1 b$ ). 


\section{B. Préparation des régimes expérimentaux}

Les régimes expérimentaux sont formulés de façon à être acceptés par les larves:

Les poudres de feuilles de luzerne sont introduites au taux de $10 \mathrm{p} .100$ dans un régime dépourvu de protéine et composé de glucose ( 90 p. 100), 4 p. 100 d'un mélange de sels minéraux adaptés aux besoins de l'insecte (mélange de Mac Collum), d'une solution de vitamines du groupe $\mathrm{B}(10 \mathrm{ml})$, de carnitine et de chlorure de zinc reconnus indispensables aux besoins des larves de T. molitor (FRAENKEL et al., 1950). Ces régimes expérimentaux sont volontairement privés de cholestérol pour mettre en évidence, de façon plus nette, l'éventuel effet des différentes teneurs en saponines des variétés de luzerne étudiées (tabl. 1). Cette privation a un effet de carence qui peut être en partie compensé pendant l'expérience par les phytostérols présents dans les feuilles de luzerne.

Les substances purifiées sont incorporées dans un régime de base composé de glucose (78 p. 100), gluten (18 p. 100), du complexe minéral et vitaminique dans les mêmes proportions que dans les régimes présentés ci-dessus et de cholestérol (1 p. 100) dont l'apport est impératif. Une carence totale en stéroïdes entraîne une très faible consommation et une chute de poids ne permettant pas d'étudier l'effet des molécules introduites.

Les saponines sont incorporées à faibles doses (tabl. 3) dans ce régime de base selon la méthode des pharmaciens galénistes qui consiste à placer au fond d'un mortier les quelques mg de la substance à étudier, $2 \mathrm{~g}$ de gluten et $0,5 \mathrm{~g}$ d'érythrosine (colorant alimentaire rouge dont nous avons vérifié l'innocuité pour les larves lors d'essais préalables), et à mélanger ces produits en ajoutant progressivement le gluten jusqu'à l'obtention d'un mélange "saponines + gluten » homogène, le témoin d'homogénéité étant la coloration rose uniforme obtenue. Ce mélange reçoit ensuite les autres ingrédients du régime.

\section{Le matériel vivant}

Cinq lots de 20 larves $d u$ ver de farine (âgées de 7 semaines) pesant environ $300 \mathrm{mg}$ chacun, sont placés, pendant 28 jours sur chacun des régimes alimentaires dans une enceinte climatisée $\left(25^{\circ} \mathrm{C}\right.$ et 80 p. 100 d'humidité relative). Température et humidité pouvant varier d'un point à l'autre de l'enceinte climatisée, nous avons adopté un dispositif d'expérimentation en blocs. Dans chaque bloc tous les régimes alimentaires sont présents et répartis au hasard. En début d'expérimentation, des échantillons des aliments préparés ainsi qu'un lot de larves semblables à celles qui sont utilisées pour l'essai sont déshydratés sous vide pendant $24 \mathrm{~h}$ à $50^{\circ} \mathrm{C}$. En fin d'expérience, régimes non consommés (et contenant les fèces) et larves sont, après pesée, traités de la même façon.

La mesure de l'activité biologique des saponines réalisée avec l'insecte T. molitor consiste à nourrir les jeunes larves avec les régimes expérimentaux contenant les substances dont on désire mesurer l'effet dépressif sur 2 critères: la quantité d'aliment sec métabolisé, ASM $=$ aliment sec fourni - (aliment sec restant + fèces) et la croissance pondérale des larves pendant 28 jours, GPS en matière sèche correspondant à la différence entre le poids sec des larves en fin d'essai et le poids sec d'un lot de larve équivalent aux lots expérimentaux.

\section{RÉSULTATS}

\section{A. Performances de l'insecte sur les régimes à base de feuilles de luzerne}

Les larves ont accepté les aliments expérimentaux préparés et, même dans les cas les plus défavorables (régimes contenant la variété italienne Marchigiana, les variétés françaises Kara et Jade, ou la variété danoise Vela), aucune des variétés n'a été refusée (tabl. 1).

Les quantités d'aliment sec métabolisé (ASM) s'échelonnent de 5,6 $\mathrm{mg}$ par larve (variété Marchigiana) à 17,2 mg par larve (variété japonaise Natsuwakaba). Ces valeurs sont faibles par rapport aux résultats habituellement obtenus sur ce type d'aliments (PraCros, 1984, 1986, 1988). Un régime " témoin », composé de farine de blé tendre additionnée du complexe minéral et vitaminique, placé dans chaque essai pour déceler d'éventuelles anomalies du comportement alimentaire des larves, montre qu'à l'origine des faibles performances de l'insecte dans cette expérience se trouvent probablement des facteurs liés aux échantillons de luzerne (les valeurs obtenues pour ASM et GPS, respectivement de 120 et de $27 \mathrm{mg}$ par larve pour le « témoin blé », étant tout à fait habituelles dans nos conditions expérimentales).

Les résultats de l'analyse de variance appliquée à la variable ASM et la comparaison des moyennes selon le test de Duncan (tabl. 1) permettent de classer les 41 variétés en fonction de leur qualité nutritionnelle pour les larves de T.molitor. Malgré la diminution globale des valeurs obtenues, 5 variétés expérimentées lors d'essais antérieurs (Lahontan, Ruro, Resis, du Puits et Lutèce) se retrouvent classées dans le même ordre (Pracros, 1984, 1986, 1988).

\section{B. Comparaison des résultats du test biologique avec les teneurs en saponines déterminées par la technique de I'HPLC}

Les teneurs en saponines, génines, acides médicagénique et luzernique des 41 variétés de luzerne ayant été déterminées par pesée puis chromatographie liquide haute performance, nous leur avons comparé les résultats du test biologique avec les larves du ver de farine par le calcul des coefficients de corrélation (tabl. 2). Dans l'ensemble, les variétés de luzerne les plus pauvres en saponines, génines et acide médicagénique se caractérisent par une valeur nutritionnelle supérieure pour l'insecte. C'est le cas pour les variétés Natsuwakaba, Lahontan, Cinna, Ruro, Kayseri et Saponex. Les coefficients de corrélation, bien que non significativement différents les uns des autres, augmentent progressivement lorsque le facteur explicatif passe de la teneur en saponines, à la teneur en génines puis au taux d'acide médicagénique. 


\section{TABLEAU 1}

Origine du matériel végétal expérimenté. Teneurs des feuilles de luzerne en saponines et én génines (exprimées en p. 100 de matière sèche) et en acides médicagénique $(A M)$ et luzernique (AL) (exprimées en p. 1000 de matière sèche) déterminées par HPLC.

Valeur nutritionnelle des 41 variétés de luzerne pour la larve de Tenebrio molitor estimée par la quantité d'aliment sec métabolisé (ASM en mg/larve) et la croissance pondérale des insectes (GPS en mg/larve). Classement des variétés en fonction du critère ASM. Les moyennes suivies d'une lettre identique ne sont pas significativement différentes au seuil de 5 p. 100 d'après le test de Duncan.

Source of experimental plant material. Saponin and genin conteni in leaves (in \% of dry material) and medicagenic (AM) and lucernic (AL) acid content (in parts per thousand of dry material) obtained by HPLC.

Nutritional value of 41 varieties of alfalfa for Tenebrio molitor larvae estimated by the dry food assimilated (ASM in mo/larvae) and the dry weight gain of insects (GPS in mg/larvae). Classification of means using the ASM measure. Two means followed by the same letter are not significantly different at the $5 \%$ error level (Duncan test).

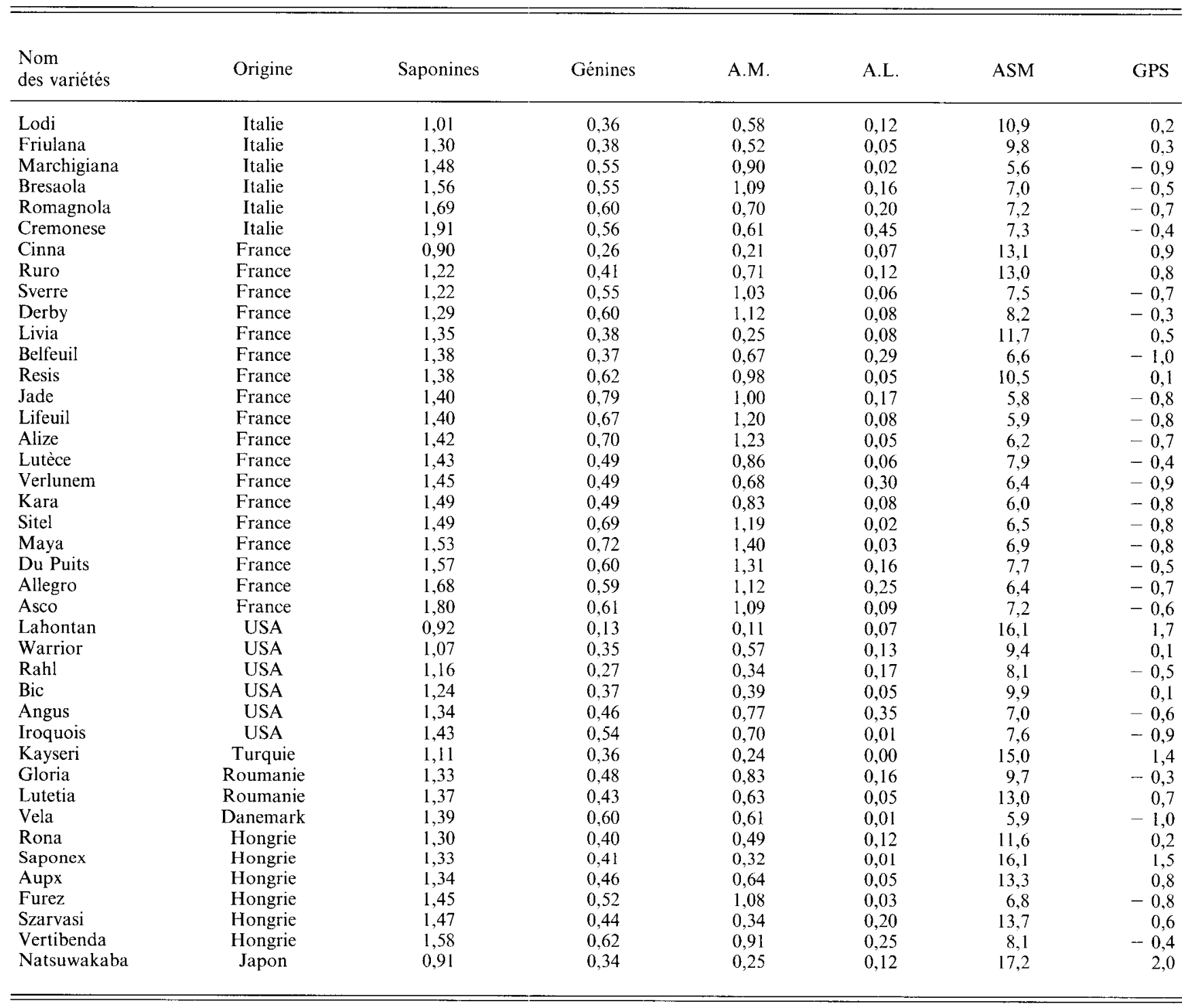

Analyse de variance appliquée à la variable ASM.

$F=14,5$ avec 40 d.d.l. l'effet variété est très hautement significatif.

( $\mathrm{F}$ table $=2,9$ au seuil de 1 p. 1000 ).

$\begin{array}{lll}\text { Marchigiana } & \text { a } & \text { Asco } \\ \text { Jade } & \text { a } & \text { Romagnola } \\ \text { Vela } & \text { a } & \text { Cremonese } \\ \text { Lifeuil } & \text { a } & \text { Sverre } \\ \text { Kara } & \text { a } & \text { Iroquois } \\ \text { Alize } & \text { a } & \text { Du Puits } \\ \text { Verlunem } & \text { a } & \text { Lutece } \\ \text { Allegro } & \text { a } & \text { Rahl } \\ \text { Sitel } & \text { a } & \text { Vertibenda } \\ \text { Belfeuil } & \text { a } & \text { Derby } \\ \text { Furez } & \text { a } & \text { Warrior } \\ \text { Maya } & \text { a } & \text { Gloria } \\ \text { Bresaola } & \text { ab } & \text { Friulana } \\ \text { Angus } & \text { ab } & \text { Bic }\end{array}$

$\begin{array}{lll}\text { abc } & \text { Resis } & \text { abcde } \\ \text { abc } & \text { Lodi } & \text { bcde } \\ \text { abc } & \text { Rona } & \text { cdef } \\ \text { abc } & \text { Livia } & \text { cdef } \\ \text { abc } & \text { Ruro } & \text { def } \\ \text { abc } & \text { Lutetia } & \text { def } \\ \text { abc } & \text { Cinna } & \text { defg } \\ \text { abc } & \text { Aupx } & \text { defg } \\ \text { abc } & \text { Szarvasi } & \text { defg } \\ \text { abcd } & \text { Kayseri } & \text { efg } \\ \text { abcd } & \text { Saponex } & \text { efg } \\ \text { abcd } & \text { Lahontan } & \text { efg } \\ \text { abcd } & \text { Natsurwakaba } & \text { g } \\ \text { abcd } & & \end{array}$


TABLEAU 2

Matrice des coefficients de corrélation entre les 6 variables étudiées. Les corrélations sont très hautement significatives (***) lorsque le coefficient de corrélation est supérieur ou égal à 0,49 ; Elles sont hautement significatives (**) lorsqu'il est supérieur à 0,39 et sont significatives (*) lorsqu'il est supérieur à 0,30 .

The matrix of carrelation coefficients between the 6 variables studied. Correlations significant at the 0.001 (***), 0.01 (**) and 0.05 (*) levels when the coefficient is greater than $0.49,0.39$ and 0.30 respectively.

\begin{tabular}{lcclll}
\hline \hline & ASM & GPS & SAP & GEN & AM \\
\hline ASM & 1 & & & & AL \\
GPS & $-0,93^{* * *}$ & 1 & & & 1 \\
SAP & $-0,62^{* * *}$ & $-0,61^{* * *}$ & 1 & 1 & $0,09 \mathrm{NS}$ \\
GEN & $-0,69^{* * *}$ & $-0,65^{* * *}$ & $0,70^{* * *}$ & $0,85^{* * *}$ & 1 \\
AM & $-0,73^{* * *}$ & $-0,70^{* * *}$ & $0,58^{* * *}$ & $0,11 \mathrm{NS}$ & $0,40^{* *}$ \\
AL & $-0,28^{*}$ & $-0,26 \mathrm{NS}$ & & & 1 \\
\hline \hline
\end{tabular}

L'équation de la droite de régression progressive multiple de la quantité d'aliment sec métabolisé (ASM) sur les teneurs en acides médicagénique (AM) et luzernique $(\mathrm{AL}): \mathrm{ASM}=15,2-6,8 \mathrm{AM}-6,8 \mathrm{AL}$ est obtenue avec un coefficient de régression très hautement significatif $(-0,75)$.

\section{Performances des larves sur les régimes contenant des saponines extraites de la luzerne et certaines de leurs fractions}

Les aliments sont bien acceptés par les insectes avec une baisse de la consommation et de la croissance pondérale liée à l'addition de saponines (tabl. 3). On a pu modéliser cet effet par l'établissement de la droite de régression de l'assimilation (ASM) sur la teneur en saponines des régimes (fig. 2). La corrélation très hautement significative obtenue entre les performances des larves et les taux de saponines, quelle que soit leur origine et leur composition, indiquent que les substances

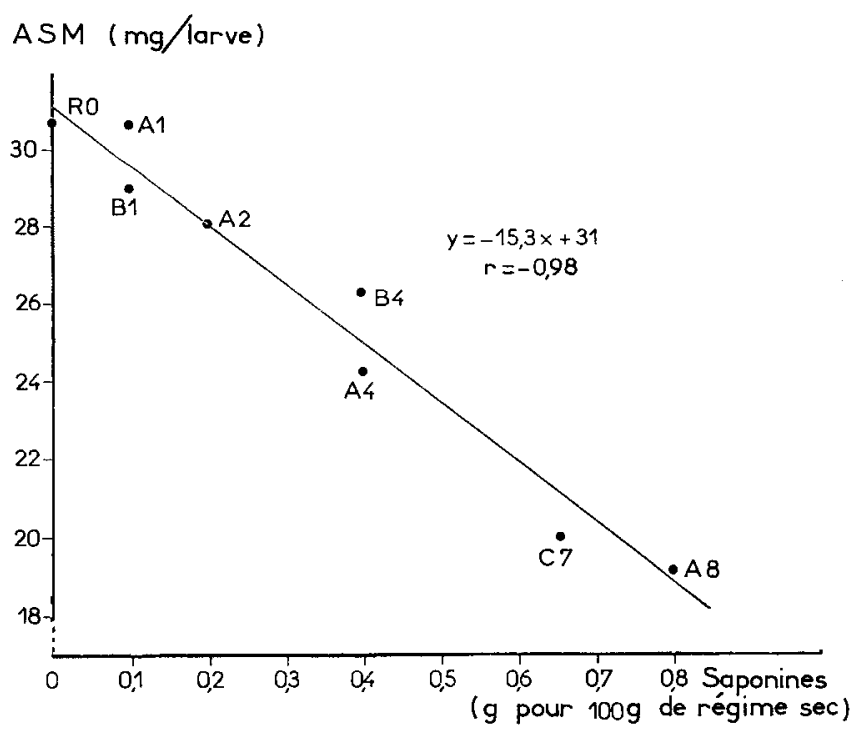

Figure 2

Régression linéaire de la quantité d'aliment sec métabolisé (ASM) sur la teneur en saponines incorporées aux régimes expérimentaux.

Regression line of dry food assimilated (ASM) on saponin levels in the experimental diets. étudiées ont conservé leur pouvoir antinutritionnel malgré les traitements qu'elles ont subi au cours de leur purification.

Une chute de la quantité d'aliment métabolisé et de la croissance est nette lorsque le taux de saponines atteint le seuil de $0,4 \mathrm{p} .100$ de régime sec (les premières différences significatives entre les moyennes des performances des larves se situant entre les régimes A4 et B4 et le régime témoin sans saponines $\mathrm{R} 0$, tabl. 3 ).

Une incorporation de $0,1 \mathrm{~g}$ de saponines dans $100 \mathrm{~g}$ de régime n'a aucun effet significatif sur les critères biologiques étudiés. Dans nos conditions expérimentales, c'est-à-dire en présence de 1 p. 100 de cholestérol, le seuil de sensibilité de la méthode se situe donc entre 0,1 et $0,2 \mathrm{p} .100$.

\section{DISCUSSION ET CONCLUSION}

Cet essai confirme les résultats déjà acquis selon lesquels la teneur en saponines de feuilles de luzerne est inversement corrélée à la valeur nutritionnelle de ces feuilles pour la larve du ver de farine. Le test «Tenebrio" peut être utilisé par les sélectionneurs de luzerne au même titre que les autres essais biologiques couramment employés (test «hémolyse » et test «Trichoderma $»)$, (PRACros, 1988).

L'étude des coefficients de corrélation entre les réponses de l'insecte et les analyses chimiques révèle un effet significatif du taux d'acide médicagénique sur la quantité d'aliment sec métabolisé $(r=-0,73)$. Le taux d'acide luzernique, non corrélé à la teneur en acide médicagénique $(r=0,09)$, ne permet pas d'expliquer les variations du critère biologique $(\mathrm{r}=-0,28)$.

Après avoir arbitrairement partagé la population de luzerne étudiée en 2 sous-populations, l'une dont la teneur en acide médicagénique est élevée (supérieure à 0,70 p. 1000 ), l'autre pauvre en cette génine (taux inférieur à 0,70 p. 1000 ), nous avons calculé les coefficients de corrélation entre le critère biologique (ASM) et les teneurs des variétés de luzerne en acides médicagénique (AM) et luzernique (AL) dans chaque sous-population (tabl. $4 \mathrm{a}$ et $4 \mathrm{~b}$ ).

Pour les 22 variétés les plus «riches» en AM, la quantité faible d'aliment sec métabolisé (la valeur moyenne d'ASM est de 7,5 avec un écart type de 1,7) 
TABLEAU 3

Origine des saponines étudiées. Teneur en saponines des régimes expérimentaux el valeur nutritionnelle pour la larve de Tenebrio molitor (ASM et GPS exprimés en mg/larve sont les movennes de 5 répétitions affectées de l'intervalle de confiance à 95 p. 100 . Deux moyennes suivies d'une même lettre ne sont pas significativement différentes au seail de 5 p. 100 , test de Duncan).

Source of saponins. Saponin content of experimental diets and nutritional value for Tenebrio molitor larvae (ASM and GPS are given in mglarvae. Results are the average of 5 replicates. The mean value is given with confidence limits at $95 \%$ confidence level. Two means followed by the same letter are not significantly different at the $5 \%$ error level, Duncan test).

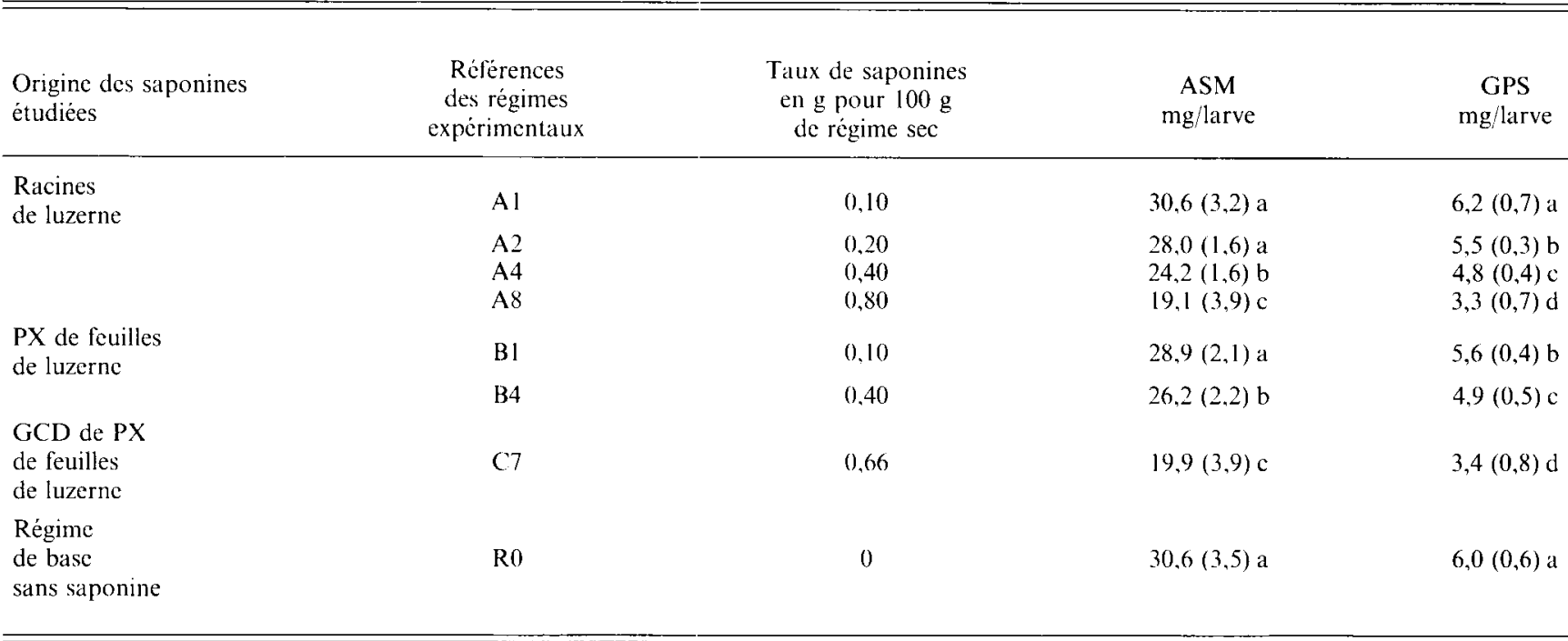

n'est corrélée ni avec la teneur en AM $(r=-0,37)$, ni avec le taux d'AL $(r=0,08)$. Tout semble se passer comme si l'on avait atteint des doses de saponines entraînant sur les insectes des effets maximums rendant impossible le classement des variétés.

Par contre, avec les 19 variétés «pauvres» en acide médicagénique, les variations d'ASM observées (valeur moyenne : $\mathrm{ASM}=11,3$ avec un écart type de 3,4), se trouvent expliquées par les teneurs en acide médicagénique (fig. 3) $(r=-0,69)$ et en acide luzernique $(r=$ $-0,51)$ et encore mieux par la prise en considération des 2 génines. L'étude de lá régression progressive

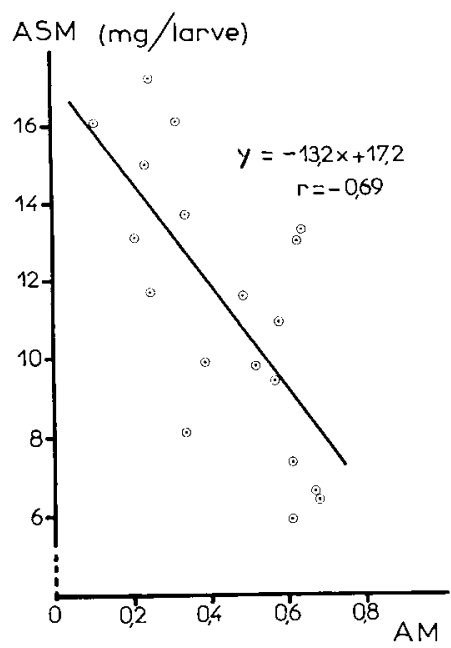

( 9 pour $1000 \mathrm{~g}$ de feuille sèche)

Figure 3

Régression linéaire de la quantité d'aliment sec métabolisé (ASM) sur la teneur en acide médicagénique (AM) des 19 variétés de haeme dont le taux en cetle génine ne dépasse pas $0.69 \mathrm{p} .1000$.

Regression line of ay food assimilated (ASM) on medicagenic acid (AM) level of 19 lucerne verieties, in which the level of this genin did not exceed $0.069 \%$. multiple d'équation $\mathrm{ASM}=17,3-11,0 \mathrm{AM}$

8,6 AL (avec un coefficient de régression de $-0,74$ ) révèle la part non négligeable de l'acide luzernique dans l'explication des effets observés (fig. 3).

Ces résultats demandent à être vérifiés. L'effet sur les larves de l'incorporation de doses croissantes de

\section{TABLEAU 4}

Matrices des coefficients de corrélation entre trois variables : la quantité d'aliment séc métaholisé (ASM), la teneur en acide médicagénique (AM) et en acide luzernique (AL) pour les 19 variétés de luzerne comtchant moins de 0.70 p. 1000 d'acide médicagénique (tabl. 4a) et pour les 22 variétés dont la quantité d'acide médicagénique dépassé ce taux (tabl. $4 b)$. Le coefficient de corrélation obtenu entre ASM et $A L$ (supérieur à (0.44) est significatif $\left(^{*}\right)$ tandis que le coefficient de corrélation entre ASM et AM (supérieur à 0,56) est hautement significatif $\left(^{* *}\right)$

Matrix of correlation coefficients between three variables: dry food assimilated (ASM), medicagenic acid content $(A M)$ and lucernic acid content $(A L)$ for the 19 varieties with level of medicagenic acid not exceeding $0.07 \%($ tabl. $4 a)$ and for the 22 varieties with level higher than $0.07 \%$ (tahl. 4h). Correlation between ASM and AL (higher than 0.44) significant at 0.05 level (*) and correlation between ASM and $A M$ (higher than 0.56) significant at 0.01 level (**).

tableau a

\begin{tabular}{lccc}
\hline \hline & ASM & AM & AL \\
\hline ASM & 1 & & \\
AM & $-0,69^{* *}$ & 1 & \\
AL & $-0,51^{*}$ & 0,37 & 1 \\
\hline
\end{tabular}

tableau b

\begin{tabular}{lccc} 
& & & \\
& ASM & AM & AL \\
\hline ASM & 1 & & \\
AM & $-0,37$ & 1 & \\
AL & 0,08 & $-0,05$ & 1 \\
& & & \\
\hline
\end{tabular}


chacune de ces 2 génines dans le régime alimentaire de base est envisagé.

L'acide médicagénique n'est donc pas le seul responsable de l'effet antinutritionnel observé et s'il est mis en évidence un effet néfaste de l'acide luzernique sur l'efficacité nutritionnelle de la luzerne pour les vertébrés, il sera peut-être intéressant de prendre en compte cette génine dans les programmes d'amélioration de la luzerne.

La méthode directe d'étude de l'action des saponines par incorporation de ces substances dans un régime de base dépourvu de luzerne et contenant 1 p. 100 de cholestérol, a permis de fixer le seuil à partir duquel les saponines perturbent de façon significative la nutrition de l'insecte. Ce seuil est d'environ 0,4 p. 100.

L'addition au régime de base du mélange des 2 saponines pures GCD 20 et GCD 21 a mis en évidence une activité antinutritionnelle de ces substances pour l'insec- te. L'extraction, la purification et l'identification des saponines se poursuivant à l'Université de Reims, le " test Tenebrio » va permettre l'étude de l'activité biologique de chacune d'entre elles.

Reçu le 22 fëvrier 1988. Accepté le 5 juillet 1988.

\section{REMERCIEMENTS}

Je remercie M. GuY (I.N.R.A., Station d'Amélioration des Plantes, 86600 Lusignan) pour nous avoir offert les échantillons de feuilles de luzerne utilisés dans cette étude, Catherine Lavaud qui a bien voulu m'autoriser à reproduire les résultats de ses analyses par HPLC et M. Massiot pour sa contribution à l'extraction des saponines et de leurs fractions, tous deux chercheurs au laboratoire de pharmacognosie de l'université de pharmacie de Reims.

\section{RÉFÉRENCES BIBLIOGRAPHIQUES}

Anderson J. O., 1957. Effect of alfalfa saponin on the performance of chicks and laying hens. Poultry Sci., 36, 873-876.

Cooney W. T., Butts S., Baco L. E., 1948. Alfalfa meal in chicks rations. Poultry Sci., 27, 828-830.

Djerassi C., Thomas D. B., Livingston A. L., Thompson C. R., 1957. Terpenoids. XXXI. The structure and stereochemistry of medicagenic acid. J. amer. chem. Soc., 76, 2271-2278.

Fraenkel G., Blewett M., Coles M., 1950. The nutrition of the mealworm Tenebrio molitor L. (Tenebrionidae, Coleoptera). Physiol. zool., 23, 92-108.

Gestetner B., 1971. Structure of a saponin from lucerne Medicago sativa. Phytochem., 10, 2221-2223.

Goldstein I. J., Hay G. W., Lewis B. A., Smith F., 1965. Controlled degradation of polysaccharides by periodate oxidation, reduction and hydrolysis. Methods Carbohydrate Chem., 5, 361-370.

Guillaume D., 1986. Les saponines des concentrés protéiques de luzerne. Thèse de docteur de l'Université de Reims, $213 \mathrm{p}$.

Heywang B. W., Thompson C. R., Kemmerer A. R., 1959. Effect of alfalfa saponin on laying chickens. Poultry Sci., 38, 268-271.
Lavaud C., 1986. Détermination de structures de saponines par R.M.N. multiimpulsionnelle. Application aux saponines des racines de luzerne. Thèse de docteur d'état ès sciences pharmaceutiques, Université de Reims, $242 \mathrm{p}$.

Livingston A. L., 1959. Lucernic acid, a new triterpen from alfalfa. $J$. org. Chem., 24, 1567.

Massiot G., Lavaud C., Guillaume D., Le Men-Olivier L., Van Binst G., 1986. Identification and sequencing of sugars in saponins using 2D IH N.M.R. Spectroscopy. J, chem. Soc., chem. Commun., 1485-1486. Pracros P., 1984. Influence de quelques substances allélochimiques sur la physiologie alimentaire des larves du ver de farine, Tenebrio molitor L. Bull. OILB/SROP, 7, 65-66.

Pracros P., 1986. Evaluation nutritionnelle du rôle des substances secondaires des plantes chez les insectes, p. 277-291. In " La nutrition des crustacés et des insectes », édit. CNERNA, Paris, $303 \mathrm{p}$.

Pracros P., 1988. Mesure de l'activité des saponines de la luzerne par les larves du ver de farine : Tenebrio molitor L. (Coléoptère, Tenebrionidae). I - Comparaison avec les résultats de divers tests biologiques. Agronomie, 8 (3), 257-263.

Rao D., Bories G., 1987. Simple gas chromatographic method for the determination of medicagenic acid in alfalfa (Medicago sativa). $J$. Chromato., 410, 169-175. 